\title{
Editorial
}

\section{Epidural analgesia in children}

The report in this issue by Wood et al. ${ }^{1}$ chronicles the development of a paediatric epidural analgesia programme at Alberta Children's Hospital. In these authors' experience, epidural analgesia was associated with good analgesia and safety, though with a considerable incidence of side effects and technical difficulties. Readers should not be put off by the quoted incidence of "complications"; many of these occurrences were anticipated and relatively minor side effects.

Recent interest in epidural analgesia in children occurs in the setting of an increased awareness of the need to provide improved pain relief for children following surgery and other forms of acute and chronic pain. Regional anaesthesia has become an important means for providing postoperative pain relief with an excellent safety and side-effect profile. ${ }^{2,3}$ Peripheral nerve blocks are especially useful for children undergoing minor procedures as outpatients, ${ }^{4}$ since these blocks provide analgesia with rapid emergence and a lower incidence of nausea than comparable analgesia produced by systemic opioids.

Single shot caudal epidural blockade with bupivacaine is useful for providing analgesia following minor procedures below the umbilicus. With proper selection of concentration (e.g., bupivacaine $0.125-0.25 \%$ ) and dose (no more than $2.5 \mathrm{mg} \cdot \mathrm{kg}^{-1}$ ), caudal blocks cause minimal interference with urination and motor function, and can be used safely for outpatients. ${ }^{5}$ For more major procedures, or for procedures involving the thorax and upper abdomen, single-shot caudal blockade using bupivacaine is less effective, and the duration of analgesia is quite short. Single-shot caudal morphine (roughly 0.03 $\mathrm{mg} \cdot \mathrm{kg}^{-1}$ for lower dermatomes or $0.05 \mathrm{mg} \cdot \mathrm{kg}^{-1}$ for thoracic dermatomes) provides more prolonged analgesia and is effective for pain arising from thoracic as well as lumbo-sacral dermatomes. ${ }^{6,7}$ Caudal analgesia with opioids is not recommended for outpatients because of a high incidence of urinary retention and a rare, but real, chance of delayed respiratory depression.

Several series suggest that epidural analgesia is particularly suitable for infants and children with respiratory

From the Department of Anesthesia, Boston Children's Hospital, Boston, Mass. disease. Although these series lack the fine detail and rigour of the corresponding adult studies by Kehlet, Rawal, and others, they suggest to this reviewer that paediatric epidural analgesia can provide outstanding analgesia and can facilitate postoperative recovery. Meignier ${ }^{8}$ first showed that thoracic epidural analgesia permitted a group of infants and children with severe pulmonary disease to undergo major thoraco-abdominal procedures with excellent recovery of pulmonary function. Ecoffey, Desparmet, Murat and their colleagues showed in a series of studies that lumbar and thoracic epidural analgesia with local anaesthetics provided excellent analgesia and haemodynamic stability. ${ }^{9-11}$ Reports by Bösenberg ${ }^{12}$ and by McNeely and others in abstract form suggest improved postoperative pulmonary function relative to historical controls in children undergoing thoracic and upper abdominal operations, respectively. Recently, Murrell and coworkers ${ }^{13}$ reported on a series of term newborns and former preterm infants undergoing primarily abdominal procedures via combined epidurallight general anaesthesia. They were able to extubate the tracheas of all infants and maintain excellent haemodynamic and respiratory stability while maintaining very low behavioural pain scores during the postoperative period. Studies by Wolf et al. ${ }^{14}$ in infants and Murat et al. in older children show that epidural analgesia suppressed hormonal-metabolic stress responses. As a corollary, while many anaesthetists omit glucose from intravenous infusions in infants undergoing general anaesthesia, this practice is more likely to produce hypoglycaemia in children under epidural-light general anaesthesia, so that in these children it is important either to administer some glucose or to monitor blood glucose concentrations to prevent hypoglycaemia. Epidural analgesia is also especially useful for children undergoing major urogenital reconstructive procedures, including ureteral reimplantation, bladder augmentation, or repair of epispadius, exstrophy or cloaca malformation sequences. Epidural analgesia can be effective in relief of postoperative bladder spasms, which can be difficult to control using standard dosing of systemic opioids. Epidural analgesia has also been used with good success for children with a variety of persistent pain syndromes, including 
cancer, ${ }^{15}$ sickle cell vaso-occlusive crisis, ${ }^{16}$ reflex sympathetic dystrophy, ${ }^{17}$ and vascular insufficiency of the feet due to meningococcal purpura fulminans. ${ }^{18}$

A learning curve is apparent in the Alberta experience, as was found in our previous report on the introduction of paediatric epidural analgesia and related regional techniques at Boston Children's Hospital from 19861989. ${ }^{19}$ For example, we found three cases of heel blisters and one case of skin breakdown at the knee in a continuous passive motion device in the first 500 patients. With increased vigilance, padding of heels and side-toside turning of patients who are confined to bed for surgical indications, we have been able to prevent these blisters subsequently, so that no heel blisters have occurred among the last 1,500 children receiving epidural analgesia at our hospital. Similarly, none of the last 1,500 children receiving epidural opioids or opioid-local anaesthetic infusions on the wards of our hospital has required naloxone or assisted ventilation for respiratory depression.

Technical problems with needles and catheters were annoying to Wood et al. and other groups share this experience. The 24-gauge paediatric catheters used by this group have been found by clinicians in many centres frequently to produce difficulties with threading of catheters, kinking, or excessive resistance to infusion, triggering pressure alarms on infusion pumps. Threading of 24 gauge catheters is more of a problem with lumbar or thoracic puncture than with caudal placement. Alternatives include: (1) use of 19-gauge short needles with 23gauge catheters, (2) use of adult-type 20-gauge catheters with short 18-gauge Tuohy needles, or (3) use of standard adult needles and catheters. Via the caudal route, standard 20-gauge adult-type epidural catheters can be advanced via either 18-gauge Tuohy needles, Crawford needles, or intravenous cannulae, according to practitioners' preferences. Disconnections and dislodgements were common as well; procedures for preventing these occurrences vary among institutions.

Most adults receive regional anaesthesia awake, permitting use of symptom-reporting to detect central nervous system toxicity with intravascular injection. Use of epinephrine-containing solutions produces heart rate increases in most, but not all, situations. Children in most settings receive regional anaesthesia while sedated or anaesthetized. This prevents use of patient reporting either for detection of intravascular injection or for confirmation of proper placement by paraesthesias or dermatomal sensory and motor blockade. In anaesthetized infants and children, epinephrine-containing solutions may not always produce a heart rate increase, particularly in patients who have not been pre-treated with anticholinergics. ${ }^{20}$ Isoproterenol may be more useful for test dosing, though more data are needed regarding neuraxial safety. ${ }^{21,22}$ Essential for safe practice of regional blockade in children is the use of fractionated dosing and constant ECG monitoring to detect other signs of intravascular effect, including ectopy and slowing as well as acceleration of the heart rate.

There is controversy regarding three related aspects of paediatric epidural analgesia: (1) the optimal choice of agents, (2) optimal site of needle puncture, and (3) the merits of cephalad advancement of epidural catheters. These issues are inter-related, and the choice of drug depends on the relationship of the catheter site to the operative site. If catheter tips can be placed at spinal levels innervating the dermatomes involved in the surgery, then I prefer combinations of dilute local anaesthetic solutions (e.g., bupivacaine $0.1 \%$ ) with opioids (e.g., fentanyl $2 \mu \mathrm{g} \cdot \mathrm{ml}^{-1}$, hydromorphone $10 \mu \mathrm{g} \cdot \mathrm{ml}^{-1}$ ) at starting infusion rates ranging from $0.2-0.3 \mu \mathrm{l} \cdot \mathrm{ml}^{-1} \cdot \mathrm{hr}^{-1}$. Dilute local anaesthetic solutions are preferable both for avoiding motor blockade (which can be distressing for many children) and for avoiding excessive dosing or insufficient spread. Conversely, if catheter tips are positioned at lumbar or caudal levels for surgery in thoracic or upper abdominal dermatomes, then hydrophilic opioids (e.g., morphine or hydromorphone) should be used.

Thoracic epidural puncture in anaesthetized children, in my view, should be restricted to: (1) patients with severe illness (e.g., chronic lung disease, cancer) undergoing extensive thoraco-abdominal surgery, and (2) practitioners who have gained considerable expertise in both adult thoracic epidural and paediatric lumbar epidural techniques. Bösenberg, Schulte-Steinberg and others, ${ }^{23}$ have advocated cephalad advancement of catheters from caudal to thoracic levels. Although this technique is generally successful in infants, it appears less reliable in older children. A study in our institution by J. Blank, C. Houck and others (manuscript submitted) suggests that there is a considerable failure rate of cephalad advancement of lumbar-to-thoracic catheters in infants and children.

For most patients, the primary rationale for epidural analgesia is to provide excellent analgesia with an acceptable side-effect profile. In order to accomplish this, proper patient selection is the key. I agree with the authors that the likelihood of urinary retention from lumbar or caudal epidural infusions is sufficiently high that the procedure should be limited primarily to patients who require bladder catheters for reasons related to the length or complexity of surgery or to young infants, who can have their bladders emptied postoperatively using the Crede manoeuvre.

The risk of infection from postoperative epidural infusions is quite rare with short-term use. A recent study 
from our hospital found no postoperative epidural infections among 1620 infusions. ${ }^{24}$

Two feared, but uncommon, complications of paediatric epidural analgesia are convulsions from local anaesthetics ${ }^{25,26}$ and respiratory depression from opioids. ${ }^{27}$ With support from the Anesthesia Patient Safety Foundation and collaboration of 15 paediatric centres worldwide, including the Alberta group, we have analyzed factors associated with these two types of adverse events among over 40,000 paediatric regional anaesthetics (manuscript submitted). These two complications were largely associated with excessive dosing. They can be made extremely rare by limitation of bupivacaine dosing to no more than $0.4-0.5 \mathrm{mg} \cdot \mathrm{kg}^{-1} \cdot \mathrm{hr}^{-1}$, by limitation of epidural morphine bolus dosing in opioid naive patients to no more than $0.05 \mathrm{mg} \cdot \mathrm{kg}^{-1}$, or by limitation of epidural morphine infusion to no more than 5 $\mu \mathrm{g} \cdot \mathrm{kg}^{-1} \cdot \mathrm{hr}^{-1}$.

Many of the management issues with a paediatric epidural programme depend on local factors related to staffing and clinical volume. It is remarkable that the group at Alberta Children's manage these patients without an anaesthetist in hospital at night. They do so by confining patients to intensive care areas and relying on acute response to emergencies by the paediatric residents and paediatric intensivists. Similar coverage exists in several $\mathrm{Ca}$ nadian hospitals, including Montreal Children's Hospital. In other institutions, where there is in-house staffing by anaesthetists, epidural analgesia is permitted in intermediate care areas or routinely on the paediatric wards. A paediatric epidural programme should not be undertaken in a haphazard or casual fashion. Systematic protocols are required to minimize errors and standardize practices. A high level of nursing and physician expertise is required.

With these cautions, and with other institutions benefiting from the learning curves of Wood et al. and other groups, epidural analgesia should become a highly effective and safe means for improving pain relief in children.

\section{L'analgésie épidurale chez l'enfant}

Le compte-rendu de Wood et al. ${ }^{1}$ publié dans ce numéro du Journal décrit l'évolution du projet d'analgésie pédiatrique par épidurale de l'Alberta Children's Hospital.
Selon l'expérience des auteurs, l'analgésie épidurale a montré son efficacité et à sa sécurité malgré une incidence considérable d'effets secondaires et de difficultés d'ordre technique. Les lecteurs ne doivent pas se laisser impressionner outre mesure par lincidence élevée des complications rapportées; plusieurs étaient anticipées et ne constituent que des effets secondaires mineurs.

Cet intérêt récent pour l'analgésie épidurale chez les enfants fait partie du contexte d'attention particulière qu'on accorde aujourd'hui au traitement de la douleur postopératoire et d'autres formes de douleur aiguë et chronique. L'anesthésie régionale est maintenant reconnue comme une technique valable de soulagement de la douleur postopératoire et jouit d'une excellente réputation au regard de la sécurité et des effets secondaires. ${ }^{2,3}$ Les blocs nerveux périphériques sont particulièrement utiles chez les enfants soumis à des interventions mineures en chirurgie ambulatoire ${ }^{4}$ grâce à un réveil rapide et à l'absence de nausées, comparativement à l'analgésie systémique aux morphiniques.

La caudale simple (une seule injection) à la bupivacaïne procure une bonne analgésie lorsque la chirurgie est mineure et située sous l'ombilic. Avec la sélection d'une concentration (par ex. bupivacaïne $0,125-0,25 \%$ ) et d'une posologie (pas plus de $2,5 \mathrm{mg} \cdot \mathrm{kg}^{-1}$ ) appropriées, la caudale gêne peu la miction et la motricité; on peut l'administrer sans inconvenients au patient ambulatoire. ${ }^{5}$ Pour les interventions plus importantes, ou celles qui intéressent le thorax et l'étage supérieur de l'abdomen, l'épidurale simple à la bupivacaine est moins efficace et la durée de l'analgésie est trop courte. Une caudale simple à la morphine (environ $0,03 \mathrm{mg} \cdot \mathrm{kg}^{-1}$ pour les métamères inférieurs ou $0,05 \mathrm{mg} \cdot \mathrm{kg}^{-1}$ pour les métamères thoraciques) procure une analgésie efficace et de plus longue durée pour la douleur d'origine thoracique ou lombosacrée. ${ }^{6,7}$ L'analgésie caudale à la morphine n'est pas recommandée en chirurgie ambulatoire à cause du pourcentage élevé de rétention urinaire et du risque minime mais réel d'une dépression respiratoire tardive.

Plusieurs études suggèrent que l'analgésie épidurale est spécialement adaptée aux nouveau-nés et enfants souffrant d'affections respiratoires. Bien que ces rapports soient peu détaillés et manquent de rigueur comparativement aux études correspondantes chez les adultes de Kehlet, Rawal et autres, elles confirment l'impression de votre analyste que l'épidurale peut procurer en pédiatrie une analgésie exceptionnelle et favoriser ainsi la récupération postchirurgicale. Meignier ${ }^{8}$ a d'abord montré que l'épidurale thoracique permettait à un groupe de nouveaunés et d'enfants souffrant d'affections pulmonaires graves de subir des interventions thoraco-abdominales majeures suivies d'une récupération profitable de la fonction respiratoire. Ecoffey, Desparmet, Murat et leurs collègues 
ont montré dans une suite d'études que l'épidurale lombaire et thoracique aux anesthésiques locaux produisait une analgésie et une stabilité hémodynamique remarquables. ${ }^{9-11}$ Les résumés des présentations de Bösenberg ${ }^{12}$ et McNeely et d'autres rapportent une amélioration de la fonction pulmonaire en postopératoire comparativement à des contrôles soumis eux-aussi à des interventions abdominales et thoraciques. Récemment, Murrell et ses associés ${ }^{13}$ ont rapporté une série de cas de nouveau-nés à terme et d'anciens prématurés soumis à des interventions abdominales sous épidurale associée à une anesthésie générale légère. Ils ont pu extuber tous les enfants rapidement et conserver une bonne stabilité hémodynamique et respiratoire avec des cotes peu élevées sur l'échelle de comportement douloureux à la période postopératoire. Les études de Wolf et al. ${ }^{14}$ et de Murat et al. chez des enfants plus âgés montrent que l'analgésie épidurale abolit les réponses hormonales et métaboliques au stress. Alors que plusieurs anesthésistes omettent d'administrer du glucose en perfusion intraveineuse aux enfants sous anesthésie générale, cette pratique est susceptible de provoquer de l'hypoglycémie sous anesthésie épidurale associée à une anesthésie générale légère; le cas échéant, il est important d'administrer du glucose ou pour le moins de monitorer la glycémie pour prévenir l'hypoglycémie. L'analgésie épidurale est particulièrement appropriée aux enfants soumis à des intervention reconstructives urogénitales majeures, telles que la réimplantation de l'uretère, l'augmentation vésicale, la réparation d'épispadias, les corrections d'exstrophie ou de malformations cloacales. L'analgésie soulage les spasmes vésicaux postopératoires quelquefois difficilement contrôlables par des doses standards de morphiniques systémiques. L'analgésie épidurale a aussi été utilisée avec succès dans une grande variété de syndromes douloureux chroniques dont le cancer, ${ }^{15}$ les crises thromboemboliques de la drépanocytose, ${ }^{16}$ les dystrophies sympathiques réflexes, ${ }^{17}$ et l'insuffisance vasculaire périphérique du syndrome de Waterhouse-Friderichsen. ${ }^{18}$

L'expérience albertaine illustre le progrès de la connaissance en analgésie pédiatrique tout comme nous l'avons montré dans notre communication plus ancienne décrivant lintroduction de l'analgésie épidurale pédiatrique et des techniques régionales connexes au Boston Children's Hospital de 1986-1989. ${ }^{19}$ Nous avions alors compté chez nos 500 premiers patients trois cas de phlyctènes du talon et un cas de rupture cutanée au genou, lésions causées par des appareils de mobilisation passive. Avec une vigilance accrue, le matelassage des talons et la mobilisation fréquente des patients alités pour des motifs chirurgicaux, nous avons été capables de prévenir les phlyctènes par la suite, de sorte qu'elles sont été totalement absentes chez les 1500 patients suivants traités sous analgésie épidurale.
De la même façon, aucun de ces 1500 patients sous perfusion épidurale aux morphiniques ou à l'association anesthésiques locaux-morphiniques n'a eu besoin de naloxone ou de ventilation artificielle pour cause de dépression respiratoire.

Des problèmes techniques d'aiguilles et de cathéters ont ennuyé Wood et al. et ils n'ont pas été les seuls. Dans plusieurs centres, le cathéter pédiatrique $24 \mathrm{G}$ a causé de difficultés avec l'enfilage dans l'aiguille, des entortillements ou une résistance extrème à l'injection avec déclenchement des alarmes de la pompe à perfusion. On rencontre plus de problèmes avec l'enfilage des cathéter $24 \mathrm{G}$ à la région lombaire et thoracique que caudale. A la place, on peut toujours utiliser: 1) les aiguilles courte $19 \mathrm{G}$ avec des cathéters $23 \mathrm{G}, 2$ ) les cathéters épiduraux pour adultes avec des aiguilles courtes Tuohy $18 \mathrm{G}$ ou 3 ) les aiguilles et cathéters standards pour adultes. Par la voie caudale, les cathéters épiduraux adultes $20 \mathrm{G}$ peuvent être avancés a travers des aiguilles Tuohy $18 \mathrm{G}$, des aiguilles Crawford ou des canules intraveineuses, selon ses préférences. Les débranchements et déplacements demeurent fréquents; les procédés de prévention de ses accidents varient d'un hôpital à un autre.

On administre l'anesthésie régionale ordinairement à des adultes éveillés ce qui permet de déceler les signes de toxicité centrale provoqués par les injections intravasculaires. L'épinéphrine ajoutée aux solutions accélère la fréquence cardiaque dans la majorité des cas mais pas dans tous. La plupart des enfants reçoivent une épidurale sous sédation ou anesthésie. Ceci empêche le patient de collaborer à la détection des injections intravasculaires - ou à la confirmation de la mise en place correcte par bloc sensoriel ou moteur. Chez les enfants anesthésiés, les solutions adrénalinées ne produisent pas toujours de la tachycardie, spécialement chez ceux qui ont déjà reçu des anticholinergiques. ${ }^{20} \mathrm{~L}$ isoprotérénol pourrait être plus utile en dose-test, mais il faut d'abord prouver son innocuité sur le système nerveux central. ${ }^{21,22}$ Pour administrer le bloc régional aux enfants en toute sécurité, il faut fractionner les doses et monitorer continuellement l'ECG la détection des autres symptômes d'injection intravasculaire, incluant l'ectopie, le ralentissement aussi bien que l'accélération du rythme cardiaque.

Trois facettes de l'analgésie épidurale pédiatrique font l'objet de débats: 1) le choix de l'agent, 2) le site de la ponction et 3) l'intérêt de pousser plus en avant les cathéters épiduraux. Ces trois facettes sont reliées entre elles et le choix de l'agent dépend de la relation entre le cathéter et le site opératoire. Si le bout du cathéter peut être avancé près des métamères du site chirurgical, je préfère des associations d'anesthésiques dilués (per ex., de la bupivacaïne $0,1 \%$ ) avec des morphiniques (par ex., fentanyl $2 \mu \mathrm{g} \cdot \mathrm{ml}^{-1}$, hdyromorphone $10 \mathrm{mg} \cdot \mathrm{ml}^{-1}$ ) avec des vi- 
tesses de perfusion initiales de $0,2-0,3 \mathrm{ml} \cdot \mathrm{kg}^{-1} \cdot \mathrm{h}^{-1}$. Les solutions étendues d'anesthésiques locaux sont préférables autant pour prévenir le bloc moteur (pénible chez les enfants) que pour éviter les doses excessives ou l'absence de progression de l'analgésie. Par contre, si l'extrémité du cathéter est positionnée au niveau lombaire ou caudal pour une chirurgie située au niveau des dermatomes thoracique ou abdominaux supérieurs, des morphiniques hydrophiles (par ex., morphine ou hydromorphone) devraient être utilisés.

A mon point de vue, la ponction épidurale thoracique chez les enfants sous anesthésie devrait être réservée 1) aux enfants gravement malades (par ex., maladie pulmonaire chronique, cancer) soumis à une chirurgie thoraco-abdominable étendue, et 2) aux anesthésistes qui ont acquis une compétence indéniable aussi bien en anesthésie épidurale thoracique qu'en anesthésie épidurale lombaire, tant chez l'enfant que chez l'adulte. Bösenberg, Schulte-Steinberg et d'autres ${ }^{23}$ ont favorisé l'ascension céphalad des cathéters du niveau caudal vers le thorax. Bien que cette technique réussisse en général chez le nourrisson, elle est moins fiable chez l'enfant plus âgé. Une étude réalisée dans notre hôpital par J. Blank, C. Houck et coll. (manuscrit soumis pour publication) porte à croire que le taux d'échec de la progression céphalad de cathéters lombaires vers la région thoracique est très élevé chez le nourrisson et l'enfant.

Pour la plupart des patients, l'analgésie épidurale a pour objectif la production d'une analgésie de qualité avec un profil acceptable d'effets secondaires. L'incidence de rétention urinaire lors des perfusions épidurales caudales ou lombaires est suffisamment élevée pour réserver la technique aux patients qui ont besoin d'une sonde urinaire pour une intervention complexe de longue durée ou aux jeunes enfants dont la vessie peut être vidée avec la manoeuvre de Crede.

A l'usage de courte durée, le risque d'infection provoquée par les perfusions épidurales postopératoire est minime. Une étude récente réalisée dans notre hôpital ne mentionne pas d'infections épidurales postopératoire après 1620 perfusions. ${ }^{24}$

Les convulsions causées par des anesthésiques locaux $^{25,26}$ et la dépression par les morphiniques ${ }^{27}$ constituent des complication redoutées de l'analgésie épidurale pédiatrique. Avec l'appui de l'Anesthesia Patient Safety Foundation et la collaboration de 15 centres pour enfants situés à travers le monde, dont le groupe albertain, nous avons analysé les facteurs associés à ces deux types d'accidents au cours de 40000 anesthésie pédiatriques régionales (manuscrit soumis pour publication). Ces deux complications sont ordinairement associées à des doses excessives. On peut les éviter presque complètement en limitant pendant la perfusion les doses de bupivacaïne à
0,4-0,5 $\mathrm{mg} \cdot \mathrm{kg}^{-1} \cdot \mathrm{h}^{-1}$, ou de morphine à $5 \mu \mathrm{g} \cdot \mathrm{kg}^{-1} \cdot \mathrm{h}^{-1}$.

Plusieurs des problèmes de gestion des programmes d'analgésie épidurale chez l'enfant sont causés par le personnel et limportance de la charge clinique. Il est remarquable que le groupe de l'Alberta Children's Hospital puisse prendre en charge ces patients sans qu'un anesthésiste soit sur place la nuit. Ils réussissent cet exploit en confinant leur patients dans des zones de soins intensifs et en s'appuyant sur la collaboration des résidents en pédiatrie et des intensivistes pédiatriques. Plusieurs couvertures identiques existent dans les hôpitaux canadiens, dont l'Hôpital pour Enfants de Montréal. Dans d'autres institutions, où des anesthésistes sont toujours disponibles sur place, l'analgésie épidurale est acceptée dans les zones de soins intermédiaires ou dans les salles du service de pédiatrie. Un projet d'épidurales pédiatriques ne doit pas être entrepris de façon désinvolte et à-peu-près. Des protocoles systématisés sont nécessaires pour minimiser les erreurs et uniformiser les pratiques. Un haut niveau de compétence médicale et infirmière est essentiel.

Avec ces réserves et avec les autres institutions profitant de l'apprentissage de Wood et al. et d'autres groupes, l'analgésie épidurale deviendra une méthode sans risque et hautement efficace permettant l'amélioration de la qualité du soulagement de la douleur chez l'enfant.

\section{References}

1 Wood CE, Goresky GV, Klassen KA, Kuwahara B, Neil SC. Complications of continuous epidural infusions for postoperative analgesia in children. Can J Anaesth 1994; 41: 613-20.

2 Sethna NF, Berde CB. Pediatric regional anesthesia. In: Gregory GA (Ed.). Pediatric Anesthesia. New York: Churchill-Livingston, 1989.

3 Dalens B. Regional anesthesia in children. Anesth Analg 1989; 68: 654-72.

4 Shandling $B$, Steward DJ. Regional analgesia for postoperative pain in pediatric outpatient surgery. J Pediatr Surg 1980; 15: 477-80.

5 Broadman LM, Hannallah RS, Norris WC, et al. Cauda anesthesia in pediatric outpatient surgery: a comparison of three different bupivacaine concentrations. Anesth Analg 1987; 66: S1-S191.

6 Krane EJ, Jacobson LE, Lynn AM, Parrot C, Tyler DC. Caudal morphine for postoperative analgesia in children: a comparison with caudal bupivacaine and intravenous morphine. Anesth Analg 1987; 66: 647-53.

7 Krane EJ, Jacobson LE, Tyler DC. Caudal epidural morphine in children: a comparison of three doses. Anesthesiology 1988; 69: A763.

8 Meignier M, Souron R, Le Neel JC. Postoperative dorsal epidural analgesia in the child with respiratory disabilities. Anesthesiology 1983; 59: 473-5. 
9 Desparmet J, Meistelman C, Barre J, Saint-Maurice C. Continuous epidural infusion of bupivacaine for postoperative pain relief in children. Anesthesiology 1987; 67: 108-10.

10 Ecoffey C, Dubousset AM, Samii K. Lumbar and thoracic epidural anesthesia for urologic and upper abdominal surgery in infants and children. Anesthesiology 1986; 65: 87-90.

11 Murat I, Delleur MM, Levy J, Esteve C, Saint-Maurice C. Continuous epidural anaesthesia for major abdominal surgery in young children. Eur $\mathrm{J}$ Anaesthesiol 1987; 4: 327-35.

12 Bösenberg AT, Hadley GP, Murray WB. Postoperative ventilation requirements following esophageal atresia repair. Journal of Pain and Symptom Management 1991; 6: 209.

13 Murrell D, Gibson PR, Cohen RC. Continuous epidural analgesia in newborn infants undergoing major surgery. $\mathrm{J}$ Pediatr Surg 1993; 28: 548-53.

14 Wolf $A R$, Eyres $R L$, Laussen $P C$, et al. Effect of extradural analgesia on stress responses to abdominal surgery in infants. Br $J$ Anaesth 1993; 70: 654-60.

15 Berde CB, Fischel N, Filardi JP, Coe CS, Grier HE, Bernstein $S C$. Caudal epidural morphine analgesia for an infant with advanced neuroblastoma: report of a case. Pain 1989; 36: 219-23.

16 Yaster M, Tobin JR, Billett C, J.F. Casella GD. Epidural. analgesia in the management of severe vaso-occlusive sickle cell crisis. Pediatrics 1994; 93: 310-5.

17 Wilder RT, Berde CB, Wolohan M, Vieyra MA, Masek $B J$, Micheli $L J$. Reflex sympathetic dystrophy in children. Clinical characteristics and follow-up of seventy patients. $J$ Bone Joint Surg Am 1992; 74: 910-9.

18 Anderson CT, Berde CB, Sethna NF, Pribaz JJ. Meningococcal purpura fulminans: treatment of vascular insufficiency in a 2-yr-old child with lumbar epidural sympathetic blockade. Anesthesiology 1989; 71: 463-4.

19 Berde CB, Sethna NF, Levin L, et al. Regional analgesia on pediatric medical and surgical wards. Intensive Care Med 1989; S40-3.

20 Desparmet J, Mateo J, Ecoffey $C$, Mazoit $X$. Efficacy of an epidural test dose in children anesthetized with halothane. Anesthesiology 1990; 72: 249-51.

21 Desparmet JF, Berde CB, Schwanz DC, Lunn RJ, Hershenson $M B$. Efficacy of adrenaline, lignocaine-adrenaline and isoprenaline as a test dose in halothane-anaesthetized lambs. Eur J Anaesthesiol 1991; 8: 123-8.

22 Perillo $M$, Sethna $N F$, Berde $C B$. Intravenous isoproterenol as a marker for epidural test-dosing in children. Anesth Analg 1993; 76: 178-81.

23 Bösenberg AT, Bland BAR, Schulte-Steinberg O, Downing $J W$. Thoracic epidural anesthesia via caudal route in infants. Anesthesiology 1988; 69: 265-9.

24 Strafford MA, Wilder RT, Berde CB. The risk of infection from epidural analgesia in children is low: a review of 1620 cases. Anesth Analg 1994 (in press).

25 McCloskey JJ, Haun SE, Deshpande JK. Bupivacaine toxicity secondary to continuous caudal epidural infusion in children. Anesth Analg 1992; 75: 287-90.

26 Agarwal R, Gutlove DP, Lockhart CH. Seizures occurring in pediatric patients receiving continuous infusion of bupivacaine. Anesth Analg 1992; 75: 284-6.

27 Valley $R D$, Bailey $A G$. Caudal morphine for postoperative analgesia in infants and children: a report of 138 cases. Anesth Analg 1991; 72: 120-4. 